\title{
Supporting Cooperative Caching in Ad Hoc Networks
}

\author{
Liangzhong Yin and Guohong Cao \\ Department of Computer Science \& Engineering \\ The Pennsylvania State University \\ E-mail: $\{$ yin, gcao\}@cse.psu.edu
}

\begin{abstract}
Most researches in ad hoc networks focus on routing, and not much work has been done on data access. A common technique used to improve the performance of data access is caching. Cooperative caching, which allows the sharing and coordination of cached data among multiple nodes, can further explore the potential of the caching techniques. Due to mobility and resource constraints of ad hoc networks, cooperative caching techniques designed for wired network may not be applicable to ad hoc networks. In this paper, we design and evaluate cooperative caching techniques to efficiently support data access in ad hoc networks. We first propose two schemes: CacheData which caches the data, and CachePath which caches the data path. After analyzing the performance of those two schemes, we propose a hybrid approach (HybridCache) which can further improve the performance by taking advantage of CacheData and CachePath while avoiding their weakness. Simulation results show that the proposed schemes can significantly reduce the query delay and message complexity when compared to other caching schemes.
\end{abstract}

Index Terms: Cooperative cache, simulations, cache management, ad hoc networks, data access.

\section{INTRODUCTION}

Wireless ad hoc networks have received considerable attention due to the potential applications in battlefield, disaster recovery, and outdoor assemblies. Ad hoc networks are ideal in situations where installing an infrastructure is not possible because the infrastructure is too expensive or too vulnerable. Due to lack of infrastructure support, each node in the network acts as a router, forwarding data packets for other nodes. Most of the previous researches [1], [2], [3], [4], [5] in ad hoc networks focus on the development of dynamic routing protocols that can efficiently find routes between two communicating nodes. Although routing is an important issue in ad hoc networks, other issue such as information (data) access is also very important since the ultimate goal of using ad hoc networks is to

This work was supported in part by the National Science Foundation (CAREER CCR-0092770 and ITR-0219711). provide information access to mobile nodes. We use the following two examples to motivate our research on data access in ad hoc networks.

Example 1: In a battlefield, an ad hoc network may consist of several commanding officers and a group of soldiers around the officers. Each officer has a relatively powerful data center, and the solders need to access the data centers to get various data such as the detailed geographic information, enemy information, and new commands. The neighboring soldiers tend to have similar missions and thus share common interests. If one soldier accessed a data item from the data center, it is quite possible that nearby soldiers access the same data some time later. It saves a large amount of battery power, bandwidth, and time if later accesses to the same data are served by the nearby soldier who has the data instead of the faraway data center.

Example 2: Recently, many mobile infostation systems have been deployed to provide information for mobile users. For example, infostations deployed by tourist information center may provide maps, pictures, history of attractive sites. Infostation deployed by a restaurant may provide menus. Due to limited radio range, an infostation can only cover a limited geographical area. If a mobile user, say Jane, moves out of the infostation range, she will not be able to access the data provided by the infostation. However, if mobile users can form an ad hoc network, these information can still be accessed by them. In such an environment, when Jane's request is forwarded to the infostation by other mobile users, it is very likely that one of the nodes along the path has already cached the requested data. Then, this node can send the data back to Jane to save time and bandwidth.

From these examples, we can see that if mobile nodes are able to work as request-forwarding routers, bandwidth and power can be saved, and delay can be reduced. Actually, cooperative caching, which allows the sharing and coordination of cached data among multiple nodes, has been widely used to improve the Web performance in wired networks. These protocols can be classified as message-based, 
directory-based, or router-based. Wessels and Claffy introduced the Internet cache protocol (ICP) [6], which has been standardized and is widely used. As a message-based protocol, ICP supports communication between caching proxies using a simple query-response dialog. Directorybased protocols such as cache digests [7] and summary cache [8] enable caching proxies to exchange information about cached content. The web cache coordination protocol [9], as a router-based protocol, transparently distributes requests among a cache array. These protocols usually assume fixed network topology and often require high computation and communication overhead. Because of resource constraints and node mobility, these techniques may not be applied directly to ad hoc networks.

In this paper, we design and evaluate cooperative caching techniques to efficiently support data access in ad hoc networks. Specifically, we propose three schemes: CachePath, CacheData and HybridCache. In CacheData, intermediate nodes cache the data to serve future requests instead of fetching data from the data center. In CachePath, mobile nodes cache the data path and use it to redirect future requests to the nearby node which has the data instead of the faraway data center. To further improve the performance, we design a hybrid approach (HybridCache), which can further improve the performance by taking advantage of CacheData and CachePath while avoiding their weakness. Simulation results show that the proposed schemes can significantly improve the performance in terms of query delay and message complexity when compared to other caching schemes.

The rest of the paper is organized as follows. In Section II, we present the CacheData scheme and the CachePath scheme. Section III presents the HybridCache scheme. The performance of the proposed schemes is evaluated in Section IV. Section V concludes the paper.

\section{Proposed Basic Cooperative Cache Schemes}

In this section, we propose two basic cooperative cache schemes and analyze their performance.

\section{A. System Model}

Fig. 1 shows part of an ad hoc network. Some nodes in the ad hoc network may have wireless interfaces to connect to the wireless infrastructure such as wireless LAN or cellular networks. Suppose node $N_{11}$ is a data source (center), which contains a database of $n$ items $d_{1}, d_{2}, \ldots, d_{n}$. Note that $N_{11}$ may be a node connecting to the wired network which has the database.

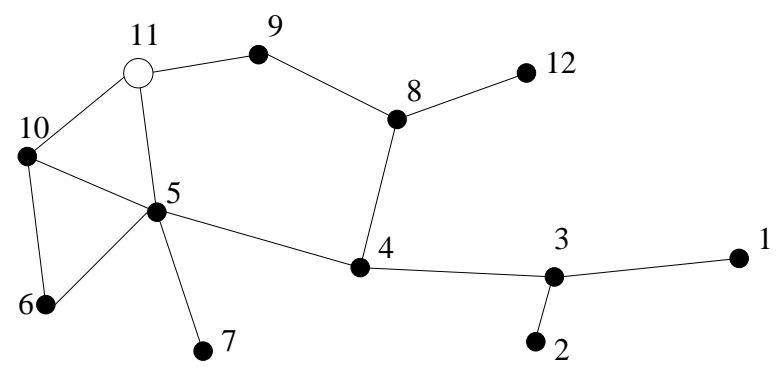

Fig. 1. an ad hoc network

In ad hoc networks, a data request is forwarded hop-byhop until it reaches the data center and then the data center sends the requested data back. Various routing algorithms have been designed to route messages in ad hoc networks. To reduce the bandwidth consumption and the query delay, the number of hops between the data center and the requester should be as small as possible. Although routing protocols can be used to achieve this goal, there is a limitation on how much they can achieve. In the following, we propose two basic cooperative caching schemes: CachePath and CacheData.

\section{B. Cache the Data (CacheData)}

In CacheData, the node caches the passing-by data locally when it finds that the data is very popular or it has enough free cache space. For example, in Fig. 1, both $N_{6}$ and $N_{7}$ request $d_{i}$ through $N_{5}, N_{5}$ knows that $d_{i}$ is popular and caches it locally. Future requests by $N_{3}, N_{4}$, or $N_{5}$ can be served by $N_{5}$ directly. Since CacheData needs extra space to save the data, it should be used prudently. Suppose the data center receives several requests for $d_{i}$ forwarded by $N_{3}$. Nodes along the path $N_{3}-N_{4}-N_{5}$ may all think that $d_{i}$ is a popular item and should be cached. However, it wastes a large amount of cache space if three of them all cache $d_{i}$. To avoid this, a conservative rule should be followed: a node does not cache the data if all requests for the data are from the same node. As in the previous example, all requests received by $N_{5}$ are from $N_{4}$, which in turn are from $N_{3}$. With the new rule, $N_{4}$ and $N_{5}$ do not cache $d_{i}$. If the requests received by $N_{3}$ are from different nodes such as $N_{1}$ and $N_{2}, N_{3}$ will cache the data. If the requests all come from $N_{1}, N_{3}$ will not cache the data, but $N_{1}$ will cache it. Certainly, if $N_{5}$ receives requests for $d_{i}$ from $N_{6}$ and $N_{7}$ later, it may also cache $d_{i}$. Note that $d_{i}$ is at least cached at the requesting node, which can use it to serve the next query.

\section{Cache the Data Path (CachePath)}

The idea of CachePath can be explained by Fig. 1. Suppose node $N_{1}$ has requested a data item $d_{i}$ from $N_{11}$. When 
$N_{3}$ forwards the data $d_{i}$ back to $N_{1}, N_{3}$ knows that $N_{1}$ has a copy of $d_{i}$. Later, if $N_{2}$ requests $d_{i}, N_{3}$ knows that the data center $N_{11}$ is three hops away whereas $N_{1}$ is only one hop away. Thus, $N_{3}$ forwards the request to $N_{1}$ instead of $N_{4}$. Note that many routing algorithms (such as AODV [10] and DSR [3]) provide the hop count information between the source and destination. By caching the data path for each data item, bandwidth and query delay can be reduced since the data can be obtained through less number of hops. However, recording the map between data items and caching nodes increases routing overhead. In the following, we propose some optimization techniques.

In CachePath, a node does not need to record the path information of all passing-by data. For example, when $d_{i}$ flows from $N_{11}$ to destination node $N_{1}$ along the path $N_{5}-$ $N_{4}-N_{3}, N_{4}$ and $N_{5}$ need not cache the path information of $d_{i}$ since $N_{4}$ and $N_{5}$ are closer to the data center than the caching node $N_{1}$. Thus, a node only records the data path when it is closer (defined later) to the caching node than the data center.

When saving the path information, a node need not save all the node information along the path. Instead, it can save only the destination node information, as the path from current router to the destination can be found by underlying routing algorithm. This can significantly reduce the path size.

Due to mobility, the node which caches the data may move. The cached data may be replaced due to the cache size limitation. As a result, the node which modified the route should reroute the request to the original data center after it finds out the problem. Thus, the cached path may not be reliable and using it may adversely increase the overhead. To deal with this issue, a node $N_{i}$ caches the data path only when the caching node, say $N_{j}$, is very close. The closeness can be defined as a function of its distance to the data center, its distance to the caching node, the route stability, and the data update rate. Intuitively, if the network is relatively stable, the data update rate is low, and its distance to the caching node (denoted as $H(i, j)$ ) is much lower than its distance to the data center (denoted as $H(i, \mathcal{C})$ ), the routing node should cache the data path. Note that $H(i, j)$ is a very important factor. If $H(i, j)$ is short, even if the cached path is broken or the data are unavailable at the caching node, the problem can be quickly detected to reduce the overhead. Certainly, $H(i, j)$ should be smaller than $H(i, \mathcal{C})$. The number of hops that a cached path can save is denoted as $H_{\text {save }}=H(i, \mathcal{C})-H(i, j)$. $H_{\text {save }}$ should be greater than a system tuning threshold, called $\mathcal{T}_{H}$, when CachePath is used.
Maintain cache consistency: There is a cache consistency issue in both CacheData and CachePath. We have done some work [11], [12] on maintaining strong cache consistency in single-hop based wireless environment. However, due to bandwidth and power constraints in ad hoc networks, it is too expensive to maintain strong cache consistency, and the weak consistency model is more attractive. A simple weak consistency model can be based on the Time-To-Live (TTL) mechanism, in which a node considers a cached copy up-to-date if its TTL has not expired, and removes the map from its routing table (or remove the cached data) if the TTL expires. As a result, future requests for this data will be forwarded to the data source.

Due to TTL expiration, some cached data may be invalidated. Usually, invalid data are removed from the cache. However, invalid data may be useful. As these data have been cached by the node, it indicates that the node is interested in these data. When a node is forwarding a data item and it finds there is an invalid copy of that data in the cache, it caches that data for future uses. To save space, when a cached data item expires, it is removed from the cache while its $i d$ is kept in "invalid" state as an indication of the node's interest. Certainly, the interest of the node may change, and the expired data should not be kept in the cache forever. In our design, if an expired data item has not been refreshed for the duration of its original TTL time (set by the data center), it is removed from the cache.

\section{Performance Analysis}

In this section, we analyze the performance of the proposed schemes. We make some assumptions to simplify the analysis to get some conclusions. The simulation results in Section IV matches the analytical results and verifies that these assumptions are reasonable.

The performance is measured by the number of hops a request is expected to travel before it reaches the data. Reducing the hop count can reduce the query delay and reduce the bandwidth and power consumption since fewer nodes are involved in the query process. Further, reducing the hop count can also reduce the workload of the data center since fewer number of requests are handled by the data center. The notations used in the analysis are as follows:

- $\bar{H}$ : the average number of hops between a mobile node and the data center.

- $P_{d d}$ : the probability that a data item is in the cache in the CacheData scheme.

- $P_{d p}$ : the probability that a data item is in the cache in the CachePath scheme.

- $P_{p p}$ : the probability that a path is in the cache in the CachePath scheme. 
- $P_{i}$ : the probability that a cached item is not usable. This may be caused by TTL expiration or broken paths because of the node movement.

- $L_{d}$ : in CacheData, the average length of the path for a request to reach the node (or the original server) which has a valid copy of the data. $L_{d}=1$ if the requester has a valid copy of the data.

- $L_{p}$ : in CachePath, the average length of the path for a request to reach the node (or the original server) which has a valid copy of data. $L_{p}=1$ if the requester has a valid copy of the data.

Given the above notations, we can obtain the expected number of hops that a request takes from node $N_{i}$ to the node which has the data. Let $P_{d}^{\prime}=P_{d d}\left(1-P_{i}\right)$, then

$$
\begin{aligned}
L_{d}= & P_{d}^{\prime} \cdot 1+\left(1-P_{d}^{\prime}\right) \cdot P_{d}^{\prime} \cdot 2+\ldots \\
& +\left(1-P_{d}^{\prime}\right) H(i, \mathcal{C})-1 \cdot P_{d}^{\prime} \cdot H(i, \mathcal{C}) \\
= & \sum_{k=1}^{H(i, \mathcal{C})}\left(1-P_{d}^{\prime}\right)^{k-1} \cdot P_{d}^{\prime} \cdot k \\
\approx & \frac{1}{P_{d}{ }^{\prime}}=\frac{1}{P_{d d}\left(1-P_{i}\right)}
\end{aligned}
$$

This equation is an approximation of $L_{d}$ since in practice $P_{d d}$ may be different at different nodes. However, Equation (1) helps us understand the effects of many important factors, and we believe the approximation is reasonable. Note that $L_{d}$ is bounded by $\bar{H}$. When $P_{d}^{\prime}$ is not too small, i.e., not less than $1 / \bar{H}$, line 4 of Equation (1) provides an adequate approximation.

To calculate $L_{p}$, three cases need to be considered:

1) data item is in the local cache.

2) a path is found in the local cache which indicates $N_{i}$ caches the requested data. Two sub-cases are possible:

(a) a valid data item is found in $N_{i}$.

(b) the data item in $N_{i}$ is not usable because of broken path or TTL expiration.

3) No data or path is found in the local cache.

Let $P_{p}^{\prime}=P_{d p}\left(1-P_{i}\right)$. The probabilities of Cases 1 , 2(a), 2(b), and 3 are $P_{p}^{\prime},\left(1-P_{p}^{\prime}\right) P_{p p}\left(1-P_{i}\right),\left(1-P_{p}^{\prime}\right) P_{p p} P_{i}$, and $\left(1-P_{p}^{\prime}\right)\left(1-P_{p p}\right)$ respectively. The number of hops needed for a request to get the data is 1 for Case 1 and $1+L_{p}$ for Case 2(a) and Case 3. For Case 2(b), the request need to travel $1+L_{p}$ to reach $N_{i}$. Then it is redirected to the data center which is $\bar{H}$ away. At last, the data item is sent back to the requester in $\bar{H}$ hops. Therefore, the average number of hops needed for the request is $\left(1+L_{p}+\bar{H}+\right.$ $\bar{H}) / 2=\bar{H}+\left(1+L_{p}\right) / 2$.

Thus

$$
\begin{aligned}
L_{p}= & P_{p}^{\prime} \cdot 1+\left(1-P_{p}^{\prime}\right) \cdot P_{p p} . \\
& \left(P_{i}\left(\bar{H}+\frac{L_{p}+1}{2}\right)+\left(1-P_{i}\right)\left(L_{p}+1\right)\right) \\
& +\left(1-P_{p}^{\prime}\right)\left(1-P_{p p}\right)\left(1+L_{p}\right)
\end{aligned}
$$

So,

$$
L_{p}=\frac{\left.P_{p}{ }^{\prime}+\left(1-P_{p}{ }^{\prime}\right) P_{p p}\left(P_{i} \bar{H}-\frac{P_{i}}{2}+1\right)\right)+\left(1-P_{p}^{\prime}\right)\left(1-P_{p p}\right)}{1-\left(1-P_{p}\right) P_{p p}\left(1-\frac{P_{i}}{2}\right)-\left(1-P_{p}^{\prime}\right)\left(1-P_{p p}\right)}
$$

In Equation (3), $P_{p p}$ is specific to CachePath. Therefore, it needs to be fixed when comparing $L_{d}$ and $L_{p}$. If $P_{p p}=0$, $L_{p}=1 /\left(P_{d p}\left(1-P_{i}\right)\right)$ and if $P_{p p}=1$,

$$
L_{p}=\frac{P_{d p}\left(1-P_{i}\right)\left(\frac{P_{i}}{2}-P_{i} \bar{H}\right)+\left(P_{i} \bar{H}-\frac{P_{i}}{2}+1\right)}{\left.1-\left(1-P_{d p}\left(1-P_{i}\right)\right)\left(1-\frac{P_{i}}{2}\right)\right)}
$$

$P_{p p}=1$ gives an upper performance bound of CachePath. Equation (4) is used in the following discussing.

Equations (1) and (4) are still complex as they contain several parameters. We can fix some parameters to get a better understanding of the relation between $L_{d}$ and $L_{p}$. Suppose $P_{i}=0$ (i.e., all the data items in the cache are valid), we have

$$
L_{d}=\frac{1}{P_{d d}} \text { and } L_{p}=\frac{1}{P_{p p}}
$$

CachePath needs less cache space to store extra data ${ }^{1}$. Therefore $P_{p p}>P_{p d}$ when the cache size is not very big, which means $L_{p}<L_{d}$.

Suppose $P_{i}=0.5$, and the cache size is big enough so that $P_{p}=P_{d}$. We obtain

$$
\begin{aligned}
L_{d} & =\frac{2}{P_{d d}} \\
L_{p} & =\frac{-2 P_{d d} \bar{H}+P_{d d}+4 \bar{H}+6}{2+3 P_{d d}}
\end{aligned}
$$

Thus,

$$
L_{d}<L_{p} \Leftrightarrow \bar{H}>\left(4-P_{d d}^{2}\right) /\left(4-2 P_{d d}^{2}\right)
$$

Note that $P_{d d} \in[0,1]$ and

$$
\frac{4-P_{d d}^{2}}{4-2 P_{d d}^{2}} \leq 1.5 \text { if } P_{d d} \in[0,1]
$$

Combining Inequalities (8) and (9) yields,

$$
L_{d}<L_{p} \text { if } \bar{H}>1.5
$$

From the above equations, we can get the following conclusions:

- Both schemes can reduce the average number of hops between the requester and the node which has the requested data. For example, when $P_{i}=0$ the number

\footnotetext{
${ }^{1}$ Note that a cached path only contains the final destination node id, as explained in Section II-C. We assume that the size of any data item is larger than the size of a data id.
} 
of hops can be reduced if the cache hit ratio is greater than $1 / \bar{H}$. If there is no cached data or path available, our schemes fall back to traditional caching scheme, where requests are sent directly to the data center.

- When the cache size is small, CachePath is better than CacheData; when the cache size is large, CacheData is better.

- When the data items are updated very slowly or mobile nodes move slowly, i.e., $P_{i}$ is small, CachePath is a good approach; while in the opposite situations, CacheData performs better.

\section{A Hybrid Caching Scheme (HybridCache)}

The performance analysis showed that CachePath and CacheData can significantly improve the system performance. We also found that CachePath performs better in some situations such as small cache size or low data update rate, while CacheData performs better in other situations. To further improve the performance, we propose a hybrid scheme HybridCache to take advantage of CacheData and CachePath while avoiding their weakness. Specifically, when a node is forwarding a data item, it caches the data or path based on some criteria. These criteria include the data item size $s_{i}$, the TTL time $T T L_{i}$, and the $H_{\text {save. }}$ For a data item $d_{i}$, the following heuristics are used to decide whether to cache data or path:

- If $s_{i}$ is small, CacheData should be adopted because the data item only needs a very small part of the cache; otherwise, CachePath should be adopted to save cache space. The threshold value for data size is denoted as $\mathcal{T}_{s}$.

- If $T T L_{i}$ is small, CachePath is not a good choice because the data item may be invalid soon. Using CachePath may result in chasing the wrong path and end up with re-sending the query to the data center. Thus, CacheData should be used in this situation. If $T T L_{i}$ is large, CachePath should be adopted. The threshold value for $T T L$ is a system tuning parameter and denoted as $\mathcal{T}_{T T L}$.

- If $H_{\text {save }}$ is large, CachePath is a good choice because it can save a large number of hops; otherwise, CacheData should be adopted to improve the performance if there is enough empty space in the cache. We adopt the threshold value $\mathcal{T}_{H}$ used in CachePath as the threshold value.

Fig. 2 shows the algorithm that apply these heuristics in HybridCache. In our design, caching a data path only needs to save a node $i d$ to the cache. This overhead is very small. Therefore, in HybridCache, when a data item $d_{i}$ is
(A) When a data item $d_{i}$ arrives:

if $\left(d_{i}\right.$ is the requested data by the current node) then cache data item $d_{i}$; return;

/* Data passing by */

if (an old version of $d_{i}$ is in the cache) then update the cached copy;

else if $\left(s_{i}<\mathcal{T}_{s}\right.$ or there is an invalid copy in the cache or there is a cached path for $d_{i}$ ) then cache data item $d_{i}$;

else if $\left(H_{\text {save }}>\mathcal{T}_{H}\right.$ and $\left.T T L_{i}>\mathcal{T}_{T T L}\right)$ then cache the path of $d_{i}$;

(B) When cache replacement is necessary:

while (not enough free space and

there are invalid data items in the cache) do Remove an invalid data item;

while (not enough free space) do /*still need space*/ Remove a valid data item;

(C) When a request for data item $d_{i}$ arrives:

if (there is a valid copy in cache) then

send $d_{i}$ to the requester;

else if (there is a valid path for $d_{i}$ in the cache) then

forward the request to the caching node; else

forward the request to the data center;

\section{Fig. 2. The hybrid caching scheme}

decided to be cached using CacheData, the path for $d_{i}$ is also cached. Later, if the cache replacement algorithm decides to remove $d_{i}$, it removes the cached data while keeping the path for $d_{i}$. From some point of view, CacheData degrades to CachePath for $d_{i}$. Similarly, CachePath can be upgraded to CacheData again when data item $d_{i}$ passes by. Comparing to Other Schemes

To effectively disseminate data in ad hoc networks, data replication and caching can be used. Data replication in ad hoc networks have been studied in [13]. However, these schemes may not be very effective due to the following reasons: First, because of frequent node movement, powering off or failure, it is hard to find stable mobile nodes to host the replicated data; Second, the cost of initial distribution of the replicated data and the cost of redistributing the data to deal with node movement or failure is very high.

Unlike data replication, caching has less overhead. In traditional caching schemes, referred to as SimpleCache in this paper, only the query node caches the received data. If another query request for the cached data comes before the cache expires, the node uses the cached data to serve the query. In case of a cache miss, it has to get the data from the data center. To utilize the caches of neighbor nodes, in the 
7DS architecture [14], users can cache data and share with neighbors when experiencing intermittent connectivity to the Internet. However, the focus of 7DS is on single-hop environment instead of multi-hop. As a result, a user only broadcasts the request to its neighbors to see if the data can be served from their caches.

A cooperative caching scheme designed specifically for accessing multimedia objects in ad hoc networks has been proposed in [15]. When a query comes, this scheme relies on flooding to find the nearest node that have the requested object. We refer this approach as the FloodCache scheme. Using flooding can reduce the query delay since the request may be served by a nearby node instead of the data center faraway. Thus, it is good for multimedia application which has strict delay requirements. Another benefit of using flooding is that multiple nodes that contain the requested data can be found. If the data size is very large, when the link to one node fails, the requester can switch to other nodes to get the rest of the requested data.

However, using flooding incurs significant message overhead. To reduce the overhead, in [15] flooding is limited to nodes that are within $k$ hops distance from the requester, where $k$ is the number of hops from the requester to the data center, but the overhead is still high. In a wireless network where nodes are uniformly distributed, on average there are $\pi k^{2}$ nodes within $k$-hops range of a mobile node. Therefore $\pi k^{2}$ messages are needed to find a data item using this method. Moreover, when one message is broadcast in the network, many neighbors will receive it. Even if the mobile node is able to identify and drop duplicated messages, each node still needs to broadcast the messages at least once to ensure full coverage. Therefore, if a node has $c$ neighbors on average, the total number of messages needs to be processed is $c \pi k^{2}$. Although the message complexity is still $O\left(k^{2}\right)$, the constant factor may be very high, especially when the network density is high.

The HybridCache scheme proposed in this paper does not use flooding. Its query delay may be higher than that of FloodCache in cases where the data is cached by some nearby nodes not along the route to the data center. However, in ad hoc networks that have limited power and bandwidth, FloodCache may not be a good choice. Because FloodCache does not make use of the passing-by data information, its performance may be limited.

When cooperative caching is used, mobile nodes need to cache data besides routing. This may involve cross-layer optimization, and it may increase the processing overhead. However, the processing delay is still very low compared to the communication delay. Since most ad hoc networks are specific to some applications, cross-layer optimization can also reduce some of the processing overhead. Considering the performance improvement, the use of cooperative cache is well justified.

\section{Performance Evaluation}

The performance evaluation includes two parts. In the first part (Section IV-B), we verify the analytical results of CacheData and CachePath, and compare them to SimpleCache and HybridCache in terms of query delay. The second part (Section IV-C) compares HybridCache to SimpleCache and FloodCache in terms of query delay and message complexity.

\section{A. The Simulation Model}

The simulation is based on $n s-2$ [16] with the CMU wireless extension. In our simulation, both the AODV routing protocol [10] and the DSDV protocol [4] have been tested as the underlying routing algorithm. Because our schemes do not rely on specific routing protocols, the results from AODV and DSDV are similar. To save space, only the results based on AODV are shown here.

The node density is changed by choosing the number of nodes between 50 and 100 in a fixed area. We assume that the wireless bandwidth is $2 \mathrm{Mb} / \mathrm{s}$, and the radio range is $250 \mathrm{~m}$.

The node movement model: We model a group of nodes moving in a $1500 \mathrm{~m} \times 320 \mathrm{~m}$ rectangle area, which is similar to the model used in [5]. The moving pattern follows the random way point movement model [17]. Initially, nodes are placed randomly in the area. Each node selects a random destination and moves toward the destination with a speed selected randomly from $\left(0 \mathrm{~m} / \mathrm{s}, v_{\max } \mathrm{m} / \mathrm{s}\right)$. After the node reaches its destination, it pauses for a period of time and repeats this movement pattern. Two $v_{\max }$ values, $2 \mathrm{~m} / \mathrm{s}$ and $20 \mathrm{~m} / \mathrm{s}$, are studied in the simulation.

The client query model: The client query model is similar to what have been used in some previous studies [12], [18]. Each node generates a single stream of read-only queries. The query generate time follows exponential distribution with mean value $T_{\text {query }}$. After a query is sent out, the node does not generate new query until the query is served. The access pattern is based on Zipf - like distribution [19], which has been frequently used ([20]) to model non-uniform distribution. In the Zipf-like distribution, the access probability of the $i^{t h}(1 \leq i \leq n)$ data item is represented as follows.

$$
P_{a_{i}}=\frac{1}{i^{\theta} \sum_{k=1}^{n} \frac{1}{k^{\theta}}}
$$


where $0 \leq \theta \leq 1$. When $\theta=1$, it is the strict Zipf distribution. When $\theta=0$, it becomes the uniform distribution. Larger $\theta$ results in more "skewed" access distribution.

The access pattern of mobile nodes can be locationdependent; that is, nodes that are around the same location tend to access similar data, such as local points of interests. To simulate this kind of access pattern, a "biased" Zipf-like access pattern is used in our simulation. In this pattern, the whole simulation area is divided into 10 (X axis) by 2 (Y axis) grids. These grids are named grid $0,1,2, \ldots 9$ in a column-wise fashion. Clients in the same grid follow the same Zipf pattern, while nodes in different grids have different offset values. For example, if the generated query should access data $i d$ according to the original Zipf-like access pattern, then in grid $i$, the new $i d$ would be $(i d+n$ $\bmod i) \bmod n$, where $n$ is the database size. This access pattern can make sure that nodes in neighboring grids have similar, although not the same, access pattern.

The server model: Two data servers: server0 and server1 are placed at the opposite corners of the rectangle area. There are $n$ data items at the server side and each server maintains half of the data items. Data items with even $i d s$ are saved at server0 and the rests are at server1. The data size is uniformly distributed between $s_{\min }$ and $s_{\max }$. The data are updated only by the server. The servers serve the requests on an FCFS (first-come-first-service) basis. When the server sends a data item to a mobile node, it sends the Time-To-Live tag along with the data. The Time-To-Live value is set exponentially with mean value $T T L$. After the TTL expires, the node has to get the new version of the data either from the server or from other nodes before serving the query.

Most system parameters are listed in Table IV-A. The second column lists the default values of these parameters. In the simulation, we may change the parameters to study the impacts of these parameters. The ranges of the parameters are listed in the third column. For each workload parameter (e.g., the mean TTL time, or the mean query generate time), the mean value of the measured data is obtained by collecting a large number of samples such that the confidence interval is reasonably small. In most cases, the $95 \%$ confidence interval for the measured data is less than $10 \%$ of the sample mean.

\section{B. Simulation Results: HybridCache}

Experiments were run using different workloads and system settings. The performance analysis presented here is designed to compare the effects of different workload parameters such as cache size, mean query generate time,
TABLE I

SIMULATION PARAMETERS

\begin{tabular}{|l|l|l|}
\hline Parameter & Default value & Range \\
\hline \hline Database size $n$ & 1000 items & \\
\hline$s_{\min }(\mathrm{KB})$ & 1 & \\
\hline$s_{\max }(\mathrm{KB})$ & 10 & \\
\hline Number of nodes & 100 & 50 to 100 \\
\hline$v_{\max }(\mathrm{m} / \mathrm{s})$ & 2 & 2,20 \\
\hline Bandwidth $(\mathrm{Mb} / \mathrm{s})$ & 2 & \\
\hline$T T L($ secs $)$ & 5000 & 200 to 10000 \\
\hline Pause time $($ secs $)$ & 300 & 0 to 300 \\
\hline Client cache size $(\mathrm{KB})$ & 800 & 100 to 1200 \\
\hline $\begin{array}{l}\text { Mean query generate } \\
\text { time } T_{q u e r y}(\mathrm{secs})\end{array}$ & 5 & 1 to 100 \\
\hline Zipf parameter $\theta$ & 0.8 & 0 to 1 \\
\hline $\mathcal{T}_{H}$ & 2 & 1 to 5 \\
\hline $\mathcal{T}_{s}\left(\%\right.$ of $\left.\left(s_{\min }+s_{\max }\right)\right)$ & $40 \%$ & $10 \%$ to $80 \%$ \\
\hline $\mathcal{T}_{T T L}($ secs $)$ & 5000 & 500 to 10000 \\
\hline
\end{tabular}

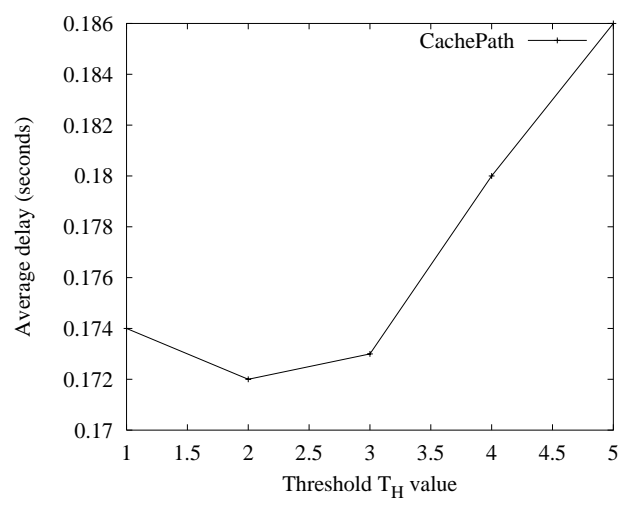

Fig. 3. Fine-tuning CachePath

node density, node mobility, and system parameters such as TTL, $\theta$ on the performance of SimpleCache, CacheData, CachePath, and HybridCache. All the schemes use the LRU algorithm for cache replacement. The effect of cache replacement algorithms is left as our future work.

1) Fine-tuning CachePath: As stated in Section II-C, the performance of CachePath is affected by the threshold value $\mathcal{T}_{H}$. A small $\mathcal{T}_{H}$ means more paths are cached, but caching too many less-valuable paths may increase the delay because the cached paths are not very reliable. A large $\mathcal{T}_{H}$ means only some valuable paths are cached. However, if $\mathcal{T}_{H}$ is too large, many paths are not cached because of the high threshold. As shown in $3, \mathcal{T}_{H}=2$ achieves a balance, and we use it in the rest of our simulations.

2) Fine-tuning HybridCache: In HybridCache, if a data item size is smaller than $\mathcal{T}_{s}$, it is cached using CacheData. If $\mathcal{T}_{s}$ is too small, HybridCache fails to identify some small but important data items; If it is too large, HybridCache caches all the data using CacheData. To find an optimal 


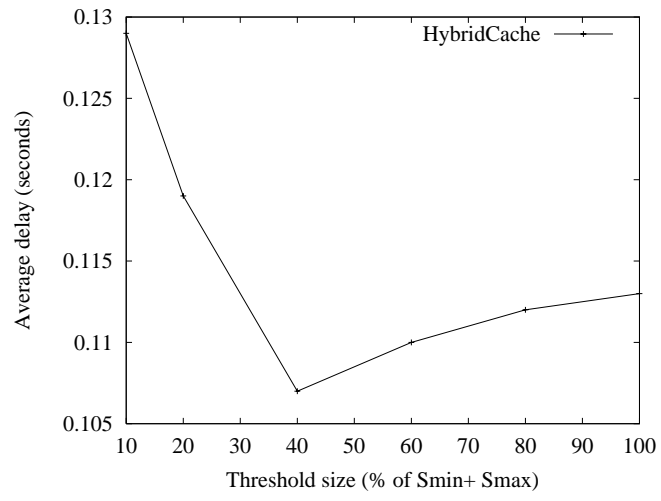

(a) Threshold $\mathcal{T}_{s}$

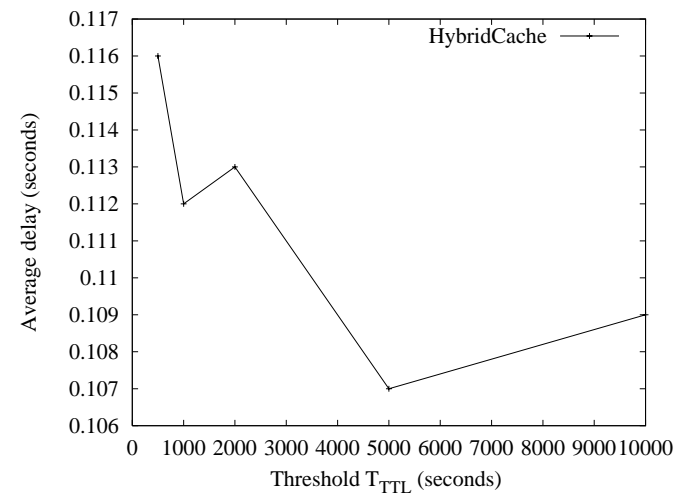

(b) Threshold $\mathcal{T}_{T T L}$

Fig. 4. Fine-tune HybridCache

value for $\mathcal{T}_{s}$, we measure the query delay as a function of $\mathcal{T}_{s}$. As $\mathcal{T}_{s}$ is related to data size, in Fig. 4 (a), we use a relative value: $\mathcal{T}_{s} /\left(S_{\min }+S_{\max }\right)$, which can give us a clearer idea of what the threshold value should be.

As shown in Fig. 4 (a), when the threshold value increases from $10 \%$ to $40 \%$, the query delay drops sharply since more data are cached. If the threshold value keeps increasing beyond $40 \%$, more passing-by data are cached, and the cache has less space to save the accessed data, and some important data may be replaced. Therefore, the delay increases. We find that a threshold value of $40 \%$ gives the best performance.

Fig. 4 (b) shows the effects of $\mathcal{T}_{T T L}$ on the average query delay. The lowest query delay is achieved when $\mathcal{T}_{T T L}=$ 5000 seconds. Compared to Fig. 4 (a), the performance difference between different $\mathcal{T}_{T T L}$ is not significant. This is because the database we studied has heterogeneous data size. Data size varies from $1 \mathrm{~KB}$ to $10 \mathrm{~KB}$. As data size is a very important factor for caching, it makes the effect of $\mathcal{T}_{T T L}$ less obvious.

3) Effects of the Cache Size: Fig. 5 shows the impacts of the cache size on the cache hit ratio and the average query delay. Cache hits can be divided into three categories: local data hit which means that the requested data is found in the local cache, remote data hit which means that the requested data is found in one of the intermediate node when the request is forwarded in the network, and path hit which means that a path is found for the request and a valid data item is found in the destination node of that path. Both remote data hit and path hit are considered as remote cache hit because the data are retrieved from remote nodes.

From Fig. 5 (a), we can see that the local hit ratio of SimpleCache is always the lowest. When the cache size is small, CacheData performs similar to SimpleCache because small cache size limits the aggressive caching of
CacheData. When the cache size is large, CacheData can cache more data for other nodes. These data can be used locally and hence the local data hit ratio increases. CachePath does not cache data for other nodes, but its cached data can be refreshed by the data passing by. Therefore, its local data hit ratio is still slightly higher than that of SimpleCache. HybridCache prefers small data items when caching data for other nodes. Therefore it can accommodate more data without wasting too much cache space, and achieve a high local data hit ratio.

Although CacheData and CachePath have similar local data hit ratio in most cases, CacheData always has higher remote data hit ratio because it caches data for other nodes. Especially when the cache size is large, more data can be cached in CacheData and its remote data hit ratio is significantly higher than that of CachePath. HybridCache has a high remote data hit ratio due to similar reason for its high local data hit ratio. Even if the path hit is not considered, HybridCache still has highest cache hit ratio in most cases. It is worth noticing that CachePath and HybridCache almost reach their highest performances when the cache size is $800 \mathrm{~KB}$. This demonstrates their low cache space requirement. This particularly shows the strength of HybridCache as it also provides the best performance at the same time.

Because of the high cache hit ratio, the proposed schemes perform much better than SimpleCache (see Fig. 5). Comparing CachePath and CacheData, when the cache size is small, CachePath has lower query delay because its path hit helps reduce the average hop count. When the cache size is greater than $800 \mathrm{~KB}$, these two schemes have similar total cache hit ratio, but CacheData has higher local data hit ratio and Remote data hit ratio. Because the hop count of local data hit is 0 and the average hop count of remote data hit is lower than that of path hit, CacheData 


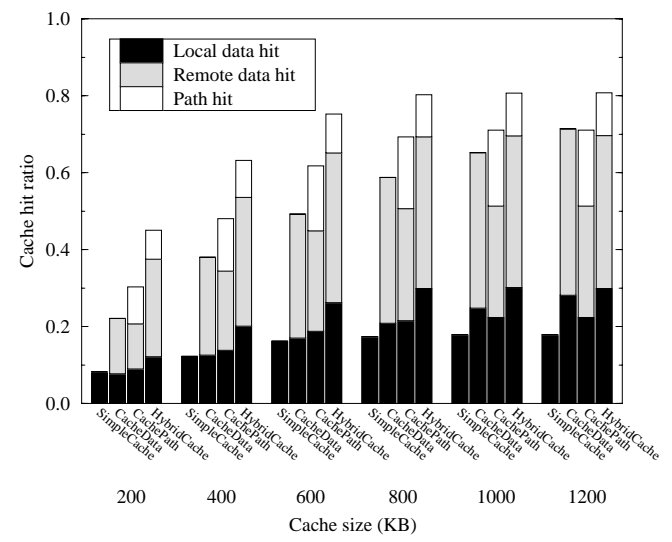

(a) Cache hit ratio

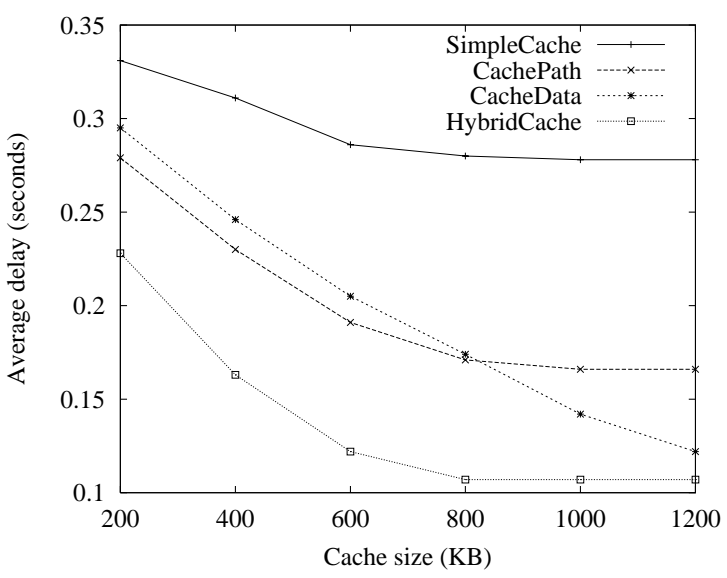

(b) Query delay

Fig. 5. The system performances as a function of the cache size

achieves low query delay. This figure also agrees with the performance comparison of CachePath and CacheData in Section IV.

Comparing these three proposed schemes, we can see that HybridCache performs much better than CacheData or CachePath, because HybridCache applies different schemes (CacheData or CachePath) to different data items, taking advantages of both CacheData and CachePath. As the result of the high local data hit ratio, remote data hit ratio and overall cache hit ratio, combined with the effect of low average hop count of remote cache hits, HybridCache achieve the best performance.

4) Effects of the Query Generate Time: Fig. 6 shows the average query delay as a function of the $T_{\text {query }}$. Both low mobility $\left(V_{\max }=2 \mathrm{~m} / \mathrm{s}\right)$ and high mobility $\left(V_{\max }=\right.$ $20 \mathrm{~m} / \mathrm{s}$ ) settings are studied. We notice that all the trends are similar except for CachePath. There are cases that CachePath even performs worse than the SimpleCache scheme. This is due to the fact that high node mobility causes more broken paths, which affects the performance of CachePath. In high mobility setting, CacheData performs better and HybridCache still performs the best in most cases.

When $T_{\text {query }}$ is small, more queries are generated and the system workload is high. As a result, the average query delay is high. As $T_{\text {query }}$ increases, less queries are generated, and the average query delay drops. If $T_{\text {query }}$ keeps increasing, the average query delay only drops slowly or even increases slightly. The reason is that the query generating speed is so low that the number of cached data is small and many cached data are not usable because their TTL have already expired before queries are generated for them. Fig. 6 verifies this trend.
Under heavy system workload ( $T_{\text {query }}$ is small), HybridCache can reduce the query delay by as much as $40 \%$ compared to CacheData or CachePath. When the system workload is extremely light, the difference between different schemes is not very large. This is because under extreme light workload, the cache hit ratio is low. Therefore, most of the queries are served by the remote data center and different schemes perform similarly.

We can also find that when the query generating speed increases ( $T_{\text {query }}$ decreases), the delay of HybridCache does not increase as fast as that of other schemes do. This demonstrates that HybridCache is less sensitive to workload increases and it can handle much heavier workload.

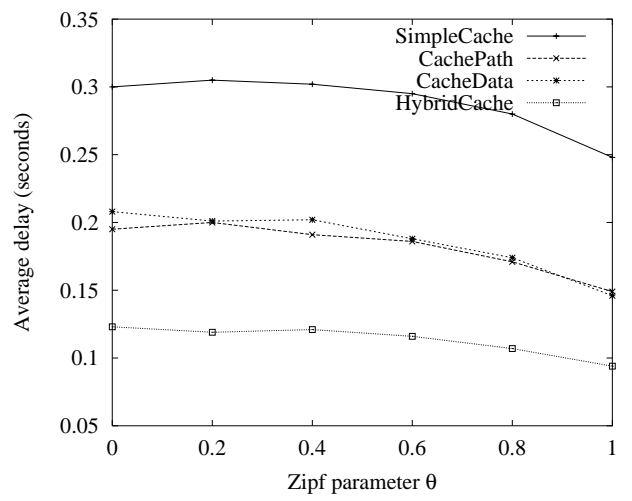

Fig. 7. The average query delay as a function of $\theta$

5) Effects of the Zipf Parameter $\theta$ : The Zipf parameter $\theta$ defines the access pattern of mobile nodes. When $\theta$ is small, the access distribution is more like a uniform distribution. The average query delay is high since the cache is not large enough to save all the data. When $\theta$ is large, the access is focused on the hot (frequently accessed) data items, and the average query delay is lower since most of 


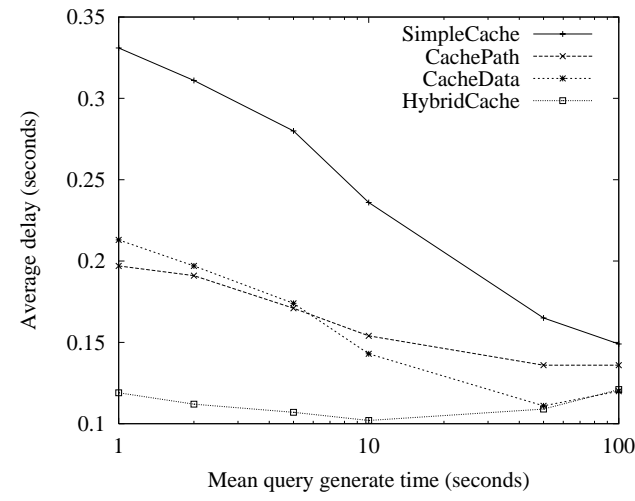

(a) $V_{\max }=2 \mathrm{~m} / \mathrm{s}$

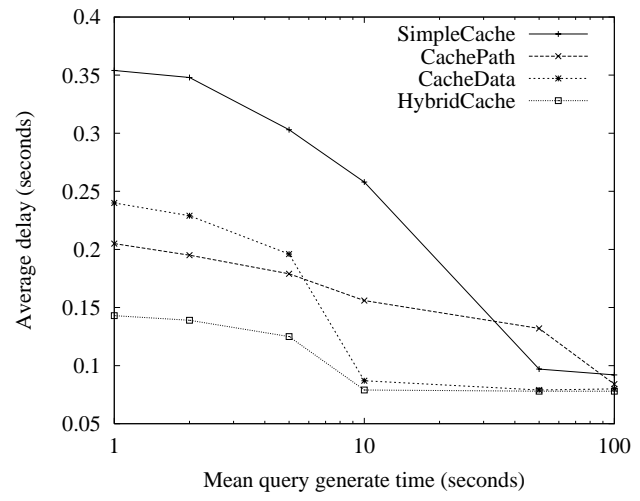

(b) $V_{\text {max }}=20 \mathrm{~m} / \mathrm{s}$

Fig. 6. The average query delay as a function of the mean query generate time $T_{\text {query }}$

these hot data can be cached. By changing $\theta$, we can see how different access patterns affect the performance. As shown in Fig. 7, our schemes perform much better than the SimpleCache scheme because the cooperation between nodes can significantly reduce the query delay.

6) Effects of TTL: Fig. 8 shows the average query delay when the $T T L$ varies from 200 seconds to 10000 seconds. TTL determines the data update rate. Higher update rate (smaller TTL) makes the cached data more likely to be invalidated, and hence the average query delay is higher. When the TTL is very small $(200 \mathrm{sec})$, all four schemes perform similarly, because most data in the cache are invalid and then the cache hit ratio is very low. Since SimpleCache does not allow nodes to cooperate with other nodes, its average query delay does not drop as fast as our schemes when TTL increases. The delay of our schemes drops much faster as TTL increases because nodes cooperate with each other to maximize the benefit of low update rate.

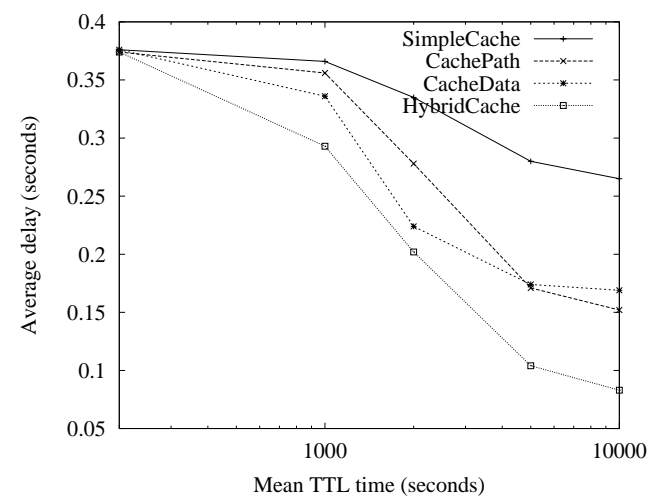

Fig. 8. The average query delay as a function of TTL

Comparing CachePath to CacheData, CacheData performs better when TTL is small, whereas CachePath performs better when TTL is big. This result again agrees with the performance analysis. HybridCache further reduces the query delay by up to $45 \%$.

7) Effects of the Node Density: Fig. 9 shows the average query delay as a function of the number of nodes in the system. As node density increases, the delay of all four schemes increase, because more nodes compete for limited bandwidth. However, our schemes increase much slower than SimpleCache. This can be explained by the fact that more data can be shared as the number of nodes increases in our schemes, which helps reduce the query delay. When the total number of nodes is small, HybridCache performs similar as CacheData and CachePath. When the number of nodes increases, HybridCache performs much better than other schemes. This indicates that HybridCache scales well with the number of nodes.

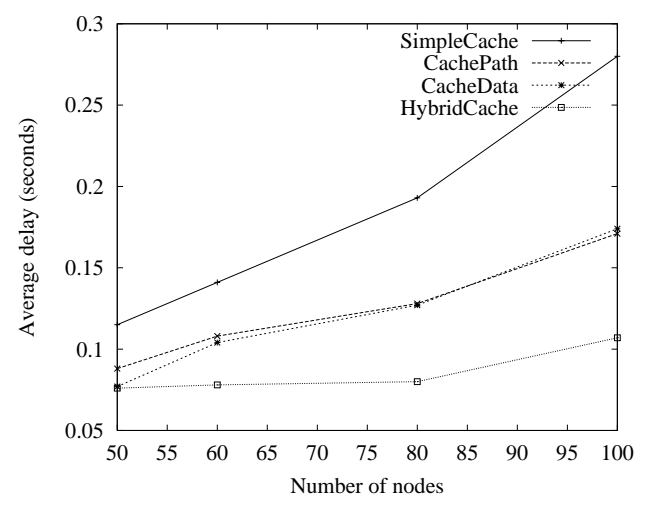

Fig. 9. The average query delay under different node density

\section{Simulation Results: Comparisons}

In this subsection, we compare the performance of the HybridCache scheme to the SimpleCache scheme and the FloodCache scheme in terms of query delay and message complexity. A commonly used message complexity metric is the total number of messages injected into the network by the query process [15]. Since each broadcast message is 
processed (received and then re-broadcasted or dropped) by every node that received it, "the number of messages processed per node" is used as the message complexity metric to reflect the efforts (battery power, CPU time, etc.) of the mobile node to deal with the messages.

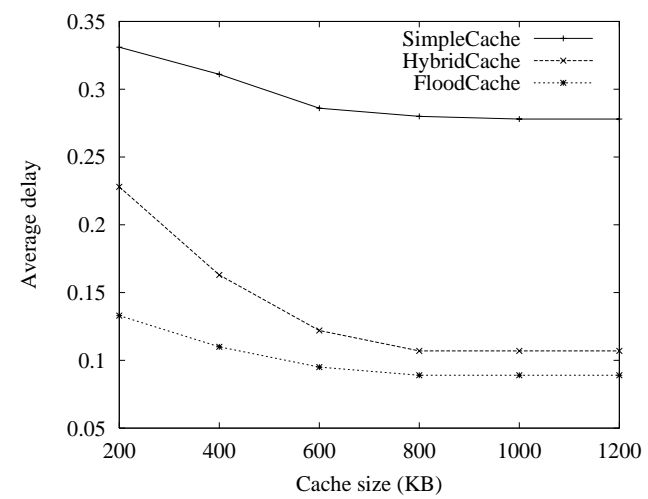

(a) Query delay

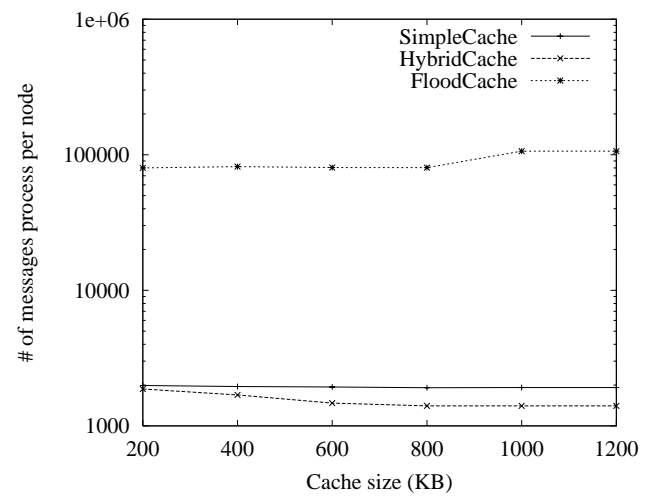

(b) Message overhead

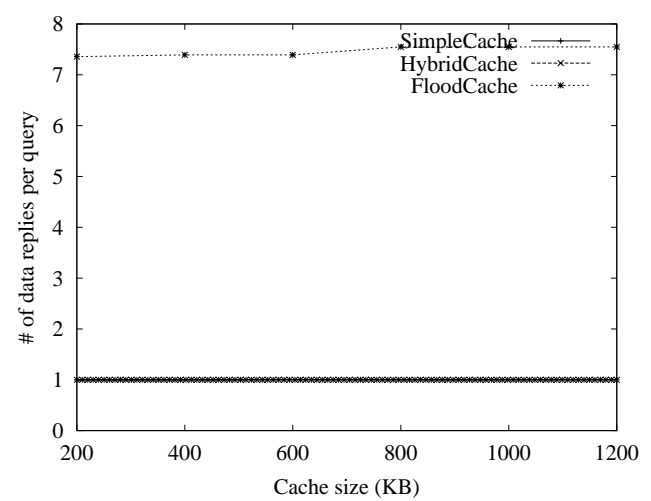

(c) \# of reply messages per query

Fig. 10. The performance as a function of the cache size

1) Effects of the Cache Size: Fig. 10 shows the impacts of the cache size on the system performance. Fig. 10 (a) shows that the query delay decreases as the cache size increases. After the cache size increases beyond $800 \mathrm{~KB}$, mobile nodes have enough cache size and the query delay does not drop significantly. The SimpleCache scheme is outperformed by cooperative caching schemes under dif- ferent cache size settings. This demonstrates that mobile nodes can benefit from sharing data with each other.

Comparing HybridCache and FloodCache, we can see that HybridCache does not perform as well as FloodCache in terms of query delay. However, Fig. 10 (b) shows that HybridCache incurs much less message overhead than FloodCache. The message overhead of HybridCache is even less than that of SimpleCache. The reason is that HybridCache gets data from nearby nodes instead of the faraway data center if possible. Therefore, the data requests and replies need to travel less number of hops and mobile nodes need to process less number of messages. As the cache size increases, the cache hit ratio of HybridCache increases and its message overhead decreases. Because FloodCache uses broadcast to find the requested data, it incurs much higher message overhead.

In FloodCache the request is sent out through flooding, and multiple copies of data replies may be returned to the requester by different nodes that have the requested data. In SimpleCache and HybridCache, this can not happen because only one request is sent out for each query in case of local cache miss. Fig. 10 (c) shows that more than 7 copies of data replies are returned per query in FloodCache. The number of duplicated data replies increases slightly as the cache size increases because data can be cached in more nodes. In our simulation, the data size is relatively small (from $1 \mathrm{~KB}$ to $10 \mathrm{~KB}$ ), and hence the duplicated messages do not affect the performance significantly. For some other environments such as multimedia accessing, transmitting duplicated data messages may waste much more power and bandwidth. As one solution, instead of sending the data to the requester upon receiving a request, mobile nodes which have the data send back an acknowledge packet. The requester can then send another unicast request to the nearest among these nodes to get the data. The drawback of this approach is that the query delay will be significantly increased.

2) Effects of the Mean Query Generate Time $T_{q u e r y}$ : HybridCache performs similar to FloodCache when $T_{\text {query }}$ is small. When the system workload is low ( $T_{\text {query }}$ is large), the difference between HybridCache and FloodCache increases. As explained in Section IV-B.4, when $T_{\text {query }}$ is small, many cached data are not usable because of the TTL expiration. Therefore, the cache hit ratio is very low. Because FloodCache can find the nearest valid data item, its query delay is small. HybridCache may not find the valid data item before a request reaches the data center. Therefore, its query delay is a little bit longer. Fig. 11 (a) shows that the query delay of FloodCache is the low- 


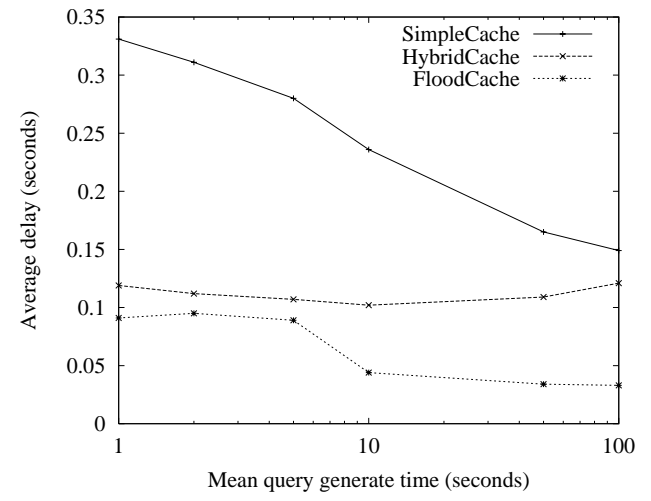

(a) Delay

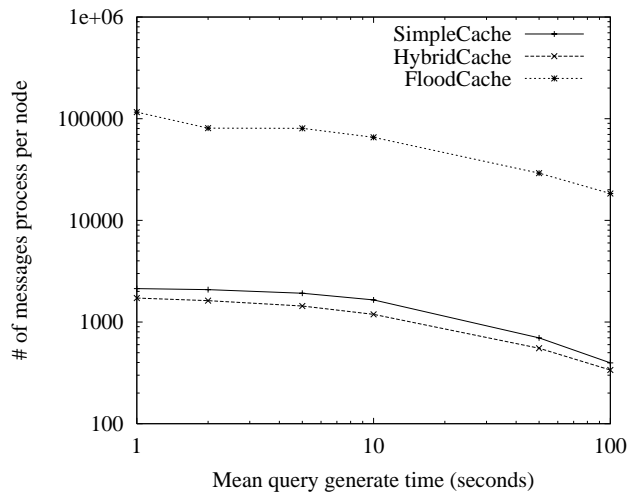

(b) Message overhead

Fig. 11. The performance as a function of the mean query generate time $T_{\text {query }}$

est. However, as can be seen from Fig. 11 (b), the message overhead of FloodCache is significantly higher than that of HybridCache.

When considering the results from both Fig. 10 and Fig. 11, we can see that FloodCache uses significantly higher message overhead to get a very minor query delay improvement over HybridCache. Thus, FloodCache may not be suitable for ad hoc networks where available bandwidth and power are scarce. HybridCache performs well because it reduces the query delay compared to SimpleCache while incurs much less overhead compared to FloodCache.

\section{CONCLUSIONS}

In this paper, we designed and evaluated cooperative caching techniques to efficiently support data access in ad hoc networks. Specifically, we proposed three schemes: CachePath, CacheData, and HybridCache. In CacheData, intermediate nodes cache the data to serve future requests instead of fetching data from the data center. In CachePath, mobile nodes cache the data path and use it to redirect future requests to the nearby node which has the data instead of the faraway data center. HybridCache takes advantage of CacheData and CachePath while avoiding their weakness. Simulation results showed that the proposed schemes can significantly reduce the query delay when compared to SimpleCache and significantly reduce the message complexity when compared to FloodCache.

\section{REFERENCES}

[1] B. Chen, K. Jamieson, H. Balakrishnan, and R. Morris, "Span: An Energy-Efficient Coordination Algorithm for Topology Maintenance in Ad Hoc Wireless Networks," ACM MobiCom, pp. 8596, July 2001.

[2] S. Das, C. Perkins, and E. Royer, "Performance comparison of two on-demand routing protocols for ad hoc networks," IEEE INFOCOM, pp. 3-12, 2000.

[3] D. Johnson and D. Maltz, "Dynamic Source Routing in Ad Hoc Wireless Network," Mobile Computing, pp. 153-181, 1996.
[4] C. Perkins and P. Bhagwat, "Highly dynamic destinationsequenced distance-vector routing (DSDV) for mobile computers," ACM SIGCOMM, pp. 234-244, 1994.

[5] Y. Xu, J. Heidemann, and D. Estrin, "Geography-informed Energy Conservation for Ad Hoc Routing," ACM MobiCom, pp. 70-84, July 2001.

[6] D. Wessels and K. Claffy, "ICP and the Squid Web Cache," IEEE Journal on Selected Areas in Communication, pp. 345-357, 1998.

[7] A. Rousskov and D. Wessels, "Web cache coordination protocol v2.0," Computer Networks and ISDN Systems, vol. 30, no. 22-23, pp. 2155-2168, 1998.

[8] L. Fan, P. Cap, J. Almeida, and A. Broder, "Summary cache: A scalable wide area web cache sharing protocol," ACM SIGCOMM, pp. 254-265, 1998.

[9] M. Cieslak, D. Foster, G. Tiwana, and R. Wilson, "Web cache coordination protocol v2.0," IETF Internet draft, 2000. http://www.ietf.org/internet-drafts/draft-wilson-wrec-wccpv2-00.txt.

[10] C. Perkins, E. Belding-Royer, and S. Das, "Ad hoc on demand distance vector (AODV) routing," Internet-Draft, draft-ietf-manetaodv-04.txt, Oct. 1999.

[11] G. Cao, "A Scalable Low-Latency Cache Invalidation Strategy for Mobile Environments," IEEE Transactions on Knowledge and Data Engineering, vol. 15, no. 5, September/October 2003 (A preliminary version appeared in ACM MobiCom'00).

[12] G. Cao, "Proactive Power-Aware Cache Management for Mobile Computing Systems," IEEE Transactions on Computer, vol. 51, no. 6, pp. 608-621, June 2002.

[13] T. Hara, "Effective Replica Allocation in Ad Hoc Networks for Improving Data Accessibility," IEEE INFOCOM, 2001.

[14] M. Papadopouli and H. Schulzrinne, "Effects of power conservation, wireless coverage and cooperation on data dissemination among mobile devices," ACM MobiHoc, Oct. 2001.

[15] W. Lau, M. Kumar, and S. Venkatesh, "A Cooperative Cache Architecture in Supporting Caching Multimedia Objects in MANETs," The Fifth International Workshop on Wireless Mobile Multimedia, 2002.

[16] ns Notes and Documentation, "http://www.isi.edu/nsnam/ns/," 2002.

[17] J. Broch, D. Maltz, D. Johnson Y. Hu, and J. Jetcheva, “A Performance Comparison of Multi-Hop Wireless Ad Hoc Network Routing Protocols," ACM MobiCom, pp. 85-97, October 1998.

[18] L. Yin, G. Cao, and Y. Cai, "A Generalized Target-driven Cache Replacement Policy for Mobile Environments," The 2003 International Symposium on Applications and the Internet, Jan 2003.

[19] G. Zipf, "Human Behavior and the Principle of Least Effort," Addison-Wesley, 1949.

[20] L. Breslau, P. Cao, L. Fan, G. Phillips, and S. Shenker, "Web Caching and Zipf-like Distributions: Evidence and Implications," IEEE INFOCOM, 1999. 